\title{
SYMMETRIES AND PROPORTIONS IN ARCHITECTURE
}

On 18 August 1418, the Florentine Arte della Lana announced an architectural competition for building the dome of Santa Maria del Fiore Cathedral using Neri di Fioravanti's design. The two main competitors were two master goldsmiths, Lorenzo Ghiberti (by whom the concept of symmetry was first explained in vulgar Italian) and Filippo Brunelleschi. Lifelong competition between the two remained sharp, but Brunelleschi received the commission, and completed the dome in 1436. It was the first "octagonal" dome in history to be built without a temporary wooden supporting frame, and was one of the most impressive projects of its time.

A thorough understanding of the physical laws and the mathematical tools for calculating stresses were centuries ahead in the future, but Brunelleschi's intuitive design marked a break with the medieval logic of construction (or "scholasticism", according to Panofsky), as well as a return to the classic Pantheon, thus, to a different concept of geometrical compactness and balance. This turnaround resulted in centuries of development and experiments on space, symmetry, scale and proportion that would give rise to 20th-century constructivism and various trends in modern and postmodern architecture. Contemporary praxis is still challenged by such experiments from minimalism to parametricism, from the complexity of space to the variability of generative structures. This provides enough reason to the architects' community for celebrating the 600th anniversary of Filippo Brunelleschi's revolutionary design for the dome of Florence Cathedral, the symbolic event which can lay claim as the birth of modern architectural thinking.

The inspiration which comes from the interdisciplinary discussions on symmetries and proportions makes it arguable whether the intent of this issue is primarily aesthetic, historical, theoretical, or rather some combination of the three. Symmetry: Culture and 
Science has never published a thematic issue about architecture before. Since it is originally a journal for sciences and cultural studies, its approach to the subject must necessarily remain holistic. However, at least to an architect, there are no other fields more closely related to symmetry as architecture, for a very simple reason: the stability or the structural integrity of a building may depend on it. Even if designers opt for an asymmetric composition, forces need to be symmetrically balanced. And still, as the modernist architect Philip Johnson has pointed out quite soundly, formal asymmetry requires a symmetrical counteract in order to remain compositionally authentic. Nonetheless, symmetry is a sign of power and control (not obviously order as it is often identified), a pattern which has never been absent from past or present societies.

If symmetry is somehow inevitable like time and space where the construction plays its ever-repeated role, then proportion is tightly connected to communication. What associates users to their buildings is not merely functionality, but the existence of something common in the human physiology and architecture. Talking about buildings, we describe capitals (heads), façades (faces), windows (eyes), wings (arms) or pedestals (feet), and summarize them in characters as if they were persons. This is not the result of theoretical anthropomorphism, but an instinct that tells the human to seek human. The human body is recognised "friendly" or "familiar" with which we can communicate, and it seems certain that this recognition is based on a canon of proportions. It is no surprise that Leonardo's illustration of the Vitruvian Man was drawn after a description in a classical architectural treatise.

Reading the papers as responses to the issue's initiative, the term "golden mean" occurs literally in almost each of them - except for only one - , so it has to be conceded that what it covers still constitutes the most common basis for comparing man to his environment. There appears to be no other common denominator so fundamental as the golden mean to link otherwise disparate disciplines, not even the Dutch Benedictine monk and architect Hans van der Laan's risky attempt to introduce a new proportional system known today as the Padovan sequence. We can also state that the Fibonacci sequence, likewise, is also in evidence.

Apart from their approach, though, it was likely to group studies according to their centres of gravity. There are attitudes which tend to analyse past and ongoing progresses historically, while others work out their answers in a practical sense. Therefore, the first and current section of Symmetries and Proportions in Architecture is a collection of reasonable papers focusing on Symmetry in History, albeit not necessarily as a yet 
concluded narrative. The second section is planned to be more than a continuation of the first, but a pile of writings introducing symmetries and proportions as crucial devices for contemporary design. Symmetry in Praxis will let us test how enduring the inevitable symmetry and the anthropomorphic proportions prove to be.

I need to say special thanks to Almantas Samalavičius, Brigitta Szilágyi, Harun Ekinoğlu, Indra McEwen, James Reese, Léon Krier and Nikos Salingaros for their suggestions and other contributions to the betterment of this pioneer issue.

Vilmos Katona

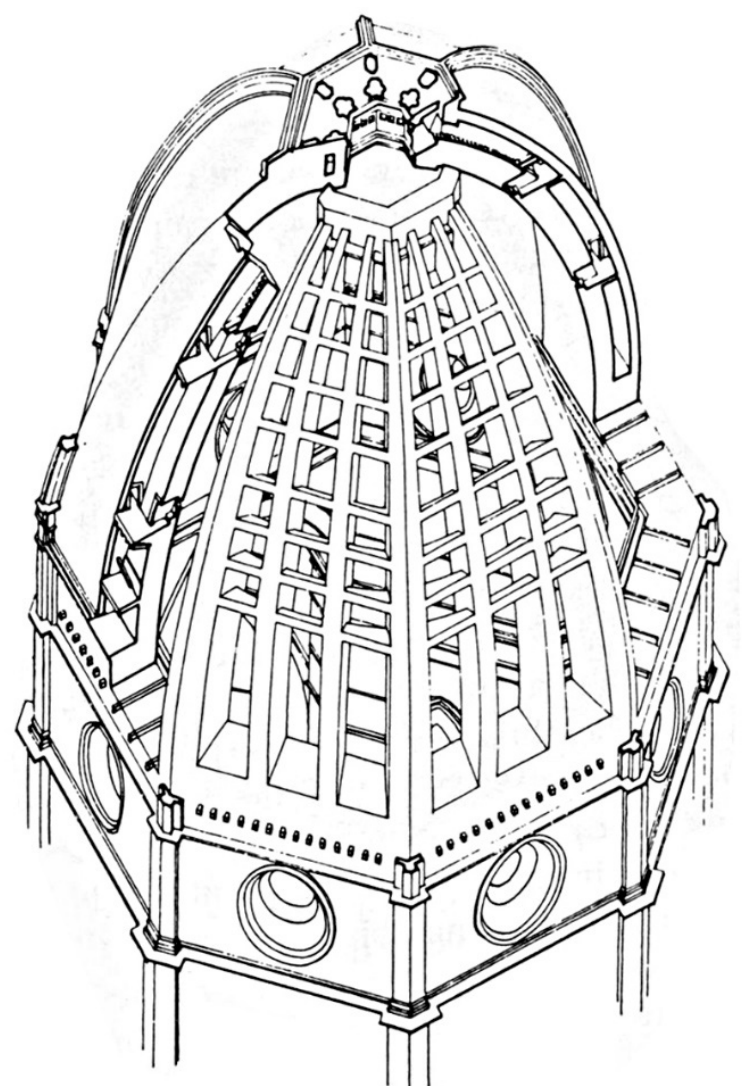

Filippo Brunelleschi's design for the construction of the dome of Santa Maria del Fiore Cathedral 